\title{
DA SISTEMA A SIMBOLO. LA LINGUA SLOVENA IN ITALIA TRA LINGUISTICA, SOCIOLOGIA E PSICOLOGIA DI MATEJKA Grgič, Marianna Kosic e Susanna PERTOT
}

Isabella Matticchio, Alpen-Adria-Universität Klagenfurt/Celovec, Austria, isabella.matticchio@aau.at

DOI: 10.31902/fII.33.2020.14

UDC: 811.163.6'27:323.15(450)

Da sistema a simbolo. La lingua slovena in Italia tra linguistica, sociologia e psicologia di Matejka Grgič, Marianna Kosic e Susanna Pertot, Prefazione di Fabiana Fusco, Roma, Aracne editrice, febbraio 2020, pp. $164 .^{1}$

II volume Da sistema a simbolo. La lingua slovena in Italia tra linguistica, sociologia e psicologia delle autrici Matejka Grgič, Marianna Kosic e Susanna Pertot con prefazione di Fabiana Fusco è stato realizzato in collaborazione con lo SLORI (Istituto sloveno di ricerche) di Trieste. Edito da Aracne editrice di Roma è disponibile sia in formato cartaceo sia in format elettronico (pdf).

Come si evince dal volume stesso, il profilo di formazione e ricerca delle tre autrici ne è garanzia di qualità: le loro competenze, la collaborazione con le istituzioni del territorio e la conoscenza della realtà della Comunità slovena hanno reso possibile la pubblicazione di un libro, il primo di questo genere, di carattere linguistico, ma dal profilo interdisciplinare, importante sia per i temi trattati nell'ambito del bi- e plurilinguismo sia per averli applicati all'esempio della Comunità nazionale slovena in Italia. Matejka Grgič è linguista e traduttrice; collabora con I'Istituto sloveno di ricerche di Trieste, il Centro di ricerche scientifiche di Capodistria e la Facoltà di lettere e filosofia dell'Università di Lubiana. Svolge ricerca prevalentemente nell'ambito della sociolinguistica, delle politiche linguistiche, dei fenomeni di contatto e delle terminologie settoriali. Marianna Kosic è psicologa con un dottorato in Politiche transfrontaliere per la vita quotidiana, è membro bilingue della comunità slovena in Italia e si interessa soprattutto di identità sociali complesse. Infine, Susanna

\footnotetext{
${ }^{1}$ Desidero ringraziare Luca Melchior per l'attenta lettura della recensione e per avermi fornito interessanti spunti di riflessione sugli argomenti trattati dalle autrici.
} 
Pertot è psicologia e psicoterapeuta nonché docente di psicolinguistica presso l'Università del Litorale di Capodistria. Si occupa di bilinguismo, educazione bilingue e di identità etnica e culturale in ambiente plurilingue.

II volume si articola in quattro capitoli tematici, ciascuno a cura dell'autrice maggiormente esperta dell'argomento che viene approfondito, seguiti dalle conclusioni e dalla rispettiva bibliografia di riferimento: Il profilo del parlante bilingue: il caso degli sloveni in Italia di Susana Pertot (21-48), Sociolinguistica e antropolinguistica delle lingue minoritarie: nuovi spunti di ricerca sulla lingua slovena in Italia di Matejka Grgič (49-83), Gli aspetti emozionali della scelta del codice linguistico. Quale lingua: prima, seconda o entrambe? di Susanna Pertot (83-124), ed infine Identità e appartenenza nella società della globalizzazione: le scelte degli sloveni in Italia di Marianna Kosic (125144). Nella prefazione dell'ordinaria Fabiana Fusco si sottolinea l'importanza del volume in quanto con lo stesso le autrici contribuiscono al "dibattito scientifico" sulle problematiche delle lingue minoritarie. Seguono l'abstract (153-156), un breve profilo delle autrici (157) e l'indice analitico (159-160).

Nell'introduzione al volume (11-15) Matejka Grgič e Susanna Pertot presentano la comunità slovena in Italia. Dopo un breve inquadramento geografico, dedicano spazio alla solida tutela giuridica della Comunità accennando qui giustamente alla diversa storia che caratterizza gli sloveni della provincia di Udine. Le autrici non scendono nei particolari, ma forniscono dei rimandi agli studi di Vidali (2011) e Vidau (2013), che possono essere utili ai lettori che desiderano approfondire l'argomento. In questa prima parte dell'Introduzione sottolineano anche la mancanza di occasioni per gli appartenenti alla Comunità slovena di usare lo sloveno, che rimane perlopiù relegato ai domini della famiglia e delle istituzioni slovene, rimarcando però al contempo la presenza di enti e associazioni il cui scopo è quello di promuovere e coltivare non soltanto la lingua, ma anche la crescita economica e culturale della Comunità. Importante in tal senso è la presenza sul territorio e il ruolo svolto (a Trieste e Gorizia) di scuole statali con lingua d'insegnamento slovena, ma anche quello dell'emittente radiotelevisiva RAl che trasmette diversi programmi radiofonici in lingua slovena, un telegiornale e dei programmi televisivi. Non menzionata dalle autrici, ma altrettanto rilevante è la collaborazione con l'emittente Radiotelevisiva Tv Koper-Capodistria (https://www.rtvslo.si/tv-capodistria) con sede in Slovenia, a KoperCapodistria, che dedica dei programmi anche agli sloveni nei paesi vicini (Italia, Austria, Ungheria e Croazia) nonché agli emigrati in altri 
continenti (https://www.rtvslo.si/tv/oddaja/173250618). Le autrici concludono questa prima sezione dell'Introduzione accennando alla situazione (socio)linguistica del territorio in cui abita la Comunità slovena, precisamente la zona orientale del Friuli Venezia Giulia, in cui convivono varietà italiane, slovene e friulane. La seconda parte dell'Introduzione contiene invece una panoramica sul volume e gli obiettivi dello stesso e la terza ed ultima, seguita dai riferimenti bibliografici, chiarisce l'uso di alcuni termini usati nel libro. Tra questi, oltre a bilinguismo e plurilinguismo appare importante la precisazione del significato del termine minoranza usato dalle autrici insieme a quello di comunità, che oggettivamente indica il rapporto numerico rispetto ad una maggioranza, ma che spesso, quando usato in ambiti formali da terzi, assume per gli appartenenti alla Comunità slovena un significato riduttivo o persino negativo visto che gli sloveni in Italia "non sono una minoranza circoscritta" e la maggior parte del popolo sloveno abita nella Repubblica di Slovenia (18). Questo aspetto è assai rilevante e fanno bene le autrici a dedicarvi la loro attenzione. Che infatti il termine minoranza possa trasmettere, per gli appartenenti, anche connotazioni negative, mostra pure la situazione della Comunità Nazionale Italiana in Croazia e Slovenia.

Il primo capitolo, scritto da Susanna Pertot, verte sul profilo del parlante bilingue (come da titolo) ed è fondamentale per poter comprendere anche il discorso dei capitoli che seguono. L'autrice si sofferma su alcuni aspetti terminologici, mostrando come concetti quali lingua materna, lingua nativa ecc. siano stati utilizzati dai diversi autori in maniera non univoca, così che anche $\mathrm{i}$ fenomeni del bilinguismo, plurilinguismo, ecc. (25-27) sono stati diversamente interpretati. Dopo questa importante premessa, l'autrice spiega come funziona l'acquisizione linguistica bilingue e plurilingue (27-29) prendendo in considerazione soprattutto i fattori che vi incidono da quello dell'età a quelli più propriamente sociali quali la motivazione e il contesto sociale che pure giocano un ruolo importante nell'apprendimento linguistico. Nella sezione successiva (29-32) Pertot discute il "vantaggio bilingue" e i vari tipi di bilinguismo che hanno origine dalle diverse dinamiche socioculturali in cui si colloca il parlante bilingue. Il padroneggiare più lingue (e avere l'occasione di parlarle entrambe!) ha come conseguenza l'interferenza linguistica (e la commutazione o alternanza di codice), fenomeno dovuto al contatto linguistico qui brevemente introdotto dall'autrice, ma trattato in modo esaustivo nel capitolo seguente da Matejka Grgič (cap. 2). La discussione dei quadri teorici di riferimento sul bilinguismo e sul parlante bi- e plurilingue nella prima parte del capitolo ne consente 
I'applicazione all'esempio dei parlanti sloveno in Italia (34-41). Pertot discute le modalità che hanno i parlanti di diventare bilingui italianosloveno e di mantenersi tali; sono cinque le possibilità descritte dall'autrice, che si rifà a un suo studio precedente, l'ultima delle quali riguarda i bambini che imparano lo sloveno fuori dal dominio della famiglia grazie alle scuole dell'infanzia oppure a quelle bilingui o slovene. Da qui, l'autrice riporta i dati di una recente ricerca condotta tra gli alunni iscritti alle scuole di ogni ordine e grado con lingua d'istruzione slovena e all'istituto bilingue di San Pietro al Natisone, che testimoniano l'aumento di genitori italiani o di lingua diversa da quella slovena (p. 36). Una situazione simile a quella riportata dall'autrice si verifica anche dall'altra parte del confine, come notato da Delton (cfr. 2016) per le scuole con lingua d'insegnamento italiana nell'Istria croata. In entrambi i casi, viene ipotizzata una correlazione tra l'entrata rispettivamente della Slovenia e della Croazia nell'Unione Europea; per cui conoscere un'altra lingua è visto come un valore aggiunto per il mercato europeo. Sarebbe dovuto alle possibilità del mercato anche lo studio dello sloveno in età adulta, che tuttavia, si ipotizza, non porterebbe al raggiungimento di un'elevata competenza linguistica visto che la maggior parte dei corsisti si ferma a corsi di livello base e intermedio. Le osservazioni si basano sul volume a cura di Čok e Jagodic (2013) citato dall'autrice che riporta i risultati di un'indagine quantitativa basata sul numero di iscrizioni ai corsi di lingua. II capitolo si conclude con alcune osservazioni sul comportamento linguistico degli sloveni nelle province di Trieste e Gorizia riportate in studi precedenti, condotti perlopiù negli anni Novanta. Pertot sottolinea però, che ci si continua ad interessare degli usi linguistici e fornisce un paio di riferimenti bibliografici recenti. Sembra, tuttavia, che questi aspetti sociolinguistici della Comunità slovena in Italia possano e debbano venir ulteriormente indagati.

Il secondo capitolo, a firma di Matejka Grgič, ha carattere socio- e antropolinguistico. Partendo dalla nozione di contatto linguistico, l'autrice spiega dapprima quali possono esserne le conseguenze, e dedica poi spazio alla situazione linguistica applicata allo sloveno (51-60), che presenta gradi di variazione tra le due comunità di parlanti, in Italia e Slovenia. Nella prima infatti, sebbene non così distante geograficamente dalla seconda, lo sloveno presenta tratti diversi, anche nella terminologia settoriale e in generale negli ambiti più formali in cui solitamente si riscontra maggiore omogeneità (p. 55-56). Questo dato riportato dall'autrice, non ulteriormente approfondito e non accompagnato da esempi, in particolare per quanto riguarda il carattere eventualmente pluricentrico dello sloveno, 
è valido anche per l'italiano parlato in Croazia, in particolare nella sfera amministrativa, che fondamentalmente è quella che richiede più omogeneità e uniformità, ma che spesso invece presenta incongruenze terminologiche anche all'interno di uno stesso o più enti regionali (si veda ad es. Lalli Paćelat et al. 2020). Grgič si sofferma anche sui concetti di erosione, deriva e folklorizzazione linguistica (60-64) della Comunità slovena in Italia ipotizzando - sulla base anche di ricerche precedenti - una possibile causa principale nell'(auto)marginalizzazione dei parlanti (cfr. 62) nei confronti della comunità nazionale di riferimento le cui ragioni andrebbero ulteriormente indagate. Questa riflessione è particolarmente utile anche per gli appartenenti alla Comunità slovena (come pure a quelli di altre Comunità minoritarie) perché rappresenta una presa di coscienza delle conseguenze di cui può risentire la lingua, che ha bisogno di venir curata e usata con costanza, a maggior ragione in territori di confine e intenso contatto linguistico, per cui la sola tutela giuridica della lingua minoritaria, a cui l'autrice dedica la sezione successiva (66-67), non è sufficiente. In merito alla riflessione di Grgič su politica e pianificazione linguistica, l'osservazione più significativa che viene fatta è la mancanza di documenti della comunità slovena in Italia per regolare e promuovere l'uso della lingua. Dopo aver sfatato il mito del primato del parlante madrelingua (67-70), l'autrice argomenta le motivazioni per cui non è possibile considerare la scuola quale fattore sufficiente all'apprendimento linguistico (70-74), anche quando l'istruzione avviene nella lingua minoritaria, come documentato da precedenti ricerche (Hickey 2001). Le ragioni sono molteplici e valgono sia per gli appartenenti alla Comunità slovena sia per gli alunni italiani: la difficoltà a raggiungere un'elevata competenza comunicativa nelle scuole minoritarie sarebbe dovuta (anche) alla presenza della lingua maggioritaria nei repertori linguistici del personale scolastico, che spesso è per molti la lingua dominante. È una buona osservazione su un aspetto potenzialmente problematico e da approfondire ulteriormente per poter sondare possibili soluzioni, ma a ciò andrebbe anche aggiunto, come sottolineato per l'appunto nella sezione successiva dall'autrice (76-81) che la vera competenza comunicativa si realizza nel quotidiano, usando la lingua fuori dal contesto scolastico e più in generale dai contesti formali, che spesso nel caso delle lingue minoritarie non avviene. Attingendo ai dati sulle realtà vicine, possiamo tracciare un parallelo con l'italiano nell'Istria croata (Scotti Jurić 2010) e slovena (Kompara 2014). Una possibile soluzione che viene proposta sarebbe quella di adottare una "strategia efficace volta soprattutto alla 'promozione della lingua' incentivando 
quanto più possibile una vera e attiva coesione sociale e culturale dell'area transfrontaliera" (83).

Susanna Pertot si occupa nel terzo capitolo degli aspetti emozionali del codice linguistico; analizza l'ipotesi che i bilingui esprimano meglio le proprie emozioni nella propria lingua natìa, ovvero nella lingua acquisita per prima. Come però emerge dalla panoramica di studi su bilinguismo ed emozioni riportati dall'autrice, se da un lato gli studi dall'approccio introspettivo sembrano testimoniare la presenza di un vantaggio emozionale della prima lingua, i risultati non sempre vengono confermati dagli studi di carattere sperimentale, a cui l'autrice dedica un'ampia sezione (100-107). Nell'attivazione emozionale, un ruolo importante verrebbe svolto anche dalla competenza linguistica nelle due lingue, per cui essa può risultare equivalente in entrambe le lingue in parlanti bilingui altamente competenti in ciascuna delle due. A risultare più accessibili nella lingua più dominante sarebbero invece le parole emozionali negative (115). Gli studi qui presentati si occupano della situazione in termini di prima e seconda lingua, ma è lecito anche chiedersi cosa succede nei bilingui simultanei precoci nati e cresciuti in un ambiente bilingue, esposti ad entrambe le lingue dalla nascita, di cui sembra che ad oggi si sappia ancora poco. Giustamente, l'autrice ricorda che - come sostenuto anche da altri ricercatori in precedenza (ad es. Pavlenko 2017) andrebbe fatta più attenzione alle variabili tradizionalmente indagate (età, contesto d'acquisizione, competenza, frequenza e contesto d'uso). ${ }^{2}$ È inoltre risaputo che queste variabili giocano sempre un ruolo importante nella descrizione del parlante bilingue e che è difficile individuare bilingui bilanciati dallo stesso profilo sociolinguistico. Nell'ultima sezione del capitolo, Pertot si sofferma sugli studi sulla lingua delle emozioni nei bilingui sloveno-italiano in Italia (110-114), che sono prevalentemente di tipo introspettivo e che fondamentalmente confermano un vantaggio emozionale del codice dominante in famiglia. Sarebbe interessante verificare se studi dello stesso tipo in altre comunità comparabili, e quindi bilingui slovenoitaliano, confermino gli stessi risultati.

Infine, il quarto ed ultimo capitolo a cura di Marianna Kosic è dedicato alla riflessione sul significato dell'identità nella società della globalizzazione. Messo in luce e discusso il complesso concetto di identità (126-127), visto come costrutto multidimensionale alla cui definizione contribuiscono contesti storici, politici, culturali, ecc., Kosic

\footnotetext{
${ }^{2}$ A queste variabili andrebbero ovviamente aggiunti anche gli aspetti culturali e le divergenze tra le due culture a cui l'individuo appartiene.
} 
riflette sui modi in cui la società della globalizzazione influenza gli aspetti identitari e il senso di appartenenza delle persone; parte dai quadri teorici di riferimento e dalle osservazioni precedentemente documentate sull'identità "etnica-nazionale-globale" per applicarle poi alla Comunità slovena in Italia. L'autrice descrive innanzitutto la società della globalizzazione e gli aspetti più problematici di quella che Zygmunt Bauman definì "modernità liquida" (128-129); definisce poi gli aspetti delle diverse generazioni (gli adulti della generazione ' $X$ ' di transizione, i millenials, la generazione della rete) (130-132); descrive i tratti dell'identità ai tempi della globalizzazione (132-135) ed infine analizza le scelte identitarie degli sloveni in Italia (135-143): come si definiscono, quali sono le loro rappresentazioni identitarie, anche in chiave contrastiva rispetto a quelle delle generazioni degli anni del dopoguerra. Quello che si registra è un concetto di identità più aperto e complesso delle generazioni più giovani, dovuto anche alla società della globalizzazione e all'educazione alla cittadinanza globale per cui, rispetto alle rappresentazioni identitarie precedenti, una singola etichetta di categorizzazione appare riduttiva.

Benché di studi sulla Comunità slovena in Italia ce ne siano, dato tra l'altro testimoniato anche nei contributi delle singole autrici che vi fanno riferimento, il volume Da sistema a simbolo. La lingua slovena in Italia tra linguistica, sociologia e psicologia rappresenta una preziosa panoramica sulla realtà linguistica della Comunità slovena in Italia, analizzata da più punti di vista - linguistico, sociologico e psicologico - con cui se ne evidenziano i punti di forza come ad esempio i vantaggi dovuti al bilinguismo, la presenza di un solido quadro giuridico di tutela linguistica e la presenza di identità ibride e più aperte, ma anche le criticità come i processi di (auto)marginalizzazione che comportano la deriva linguistica. II contributo originale delle autrici però non consiste soltanto nel far luce su questi aspetti, ma anche e soprattutto nella proposta di possibili soluzioni da adottare. Le introduzioni teoriche ai capitoli e la delucidazione terminologica rendono il testo scorrevole e ne consentono la comprensione anche al lettore non specialista. Per queste ragioni il volume rappresenta un prezioso contributo non soltanto per i ricercatori che si occupano di bi- e plurilinguismo e di lingue minoritarie, ma anche per gli appartenenti alla Comunità slovena in Italia e più in generale ad altre Comunità nazionali fuori dai confini della propria comunità di riferimento, come è il caso della Comunità Nazionale Italiana in Slovenia e Croazia. Ci auguriamo dunque, che il volume abbia la diffusione che merita, sia in ambito accademico sia nelle comunità minoritarie. 


\section{Bibliografia}

Delton, Paola. “Contributo per un'analisi delle specificità della scuola italiana in Croazia con un riferimento particolare alla pratica traduttiva del settore scolastico". Ricerche sociali 23 (2016): 5-26.

Hickey, Tina. 2001. "Mixing beginners and native speakers in minority language immersion: Who is immersing whom?". Canadian Modern Language Review, 57-3 (2001): 443-474.

Kompara, Mojca. "Je slovenska Istra še dvojezična". Jezikoslovni zapiski 20 (2014): 89-106.

Lalli Paćelat, Ivana, Brkić Bakarić, Marija e Matticchio Isabella "Službena dvojezičnost u Istarskoj županiji: stanje i perspektive." Rasprave: Časopis Instituta za hrvatski jezik i jezikoslovlje 46-2 (2020):

815-837. https://doi.org/10.31724/rihii.46.2.20.

Scotti Jurić, Rita. "Cosa resta dell'identità linguistica italiana in Istria." Adriatico/Jadran. Rivista di cultura tra le due sponde 1-2 (2010): 374-386.

Pavlenko, Aneta. "Do you wish to waive your rights? Affect and decision-making in multilingual speakers". Current opinion in Psychology 17 (2017): 74-78.

Vidali, Zaira. 2011. The Socio-Political and Ideological-Cultural Elements of the Italian Nation and the National Minority Question in Italy. Razprave in gradivo-Inštitut za narodnostna vprašanja 65 (1990): 36-59.

Vidau, Zaira. The Legal Protection of national and Linguistic Minorities in the region of Friuli Venezia Giulia: A comparison of the Three regional Laws for the "Slovene Linguistic Minority", for the "Friulian Language" and for the "German-Speaking Minorities". Treatises and Documents, Journal of Ethnic Studies 71 (2013): 27-52. 